\title{
Linear Clutter Removal from Urban Panoramas
}

\author{
Mahsa Kamali ${ }^{1}$, Eyal Ofek ${ }^{2}$, Forrest Iandola ${ }^{1}$, Ido Omer ${ }^{2}$, and John C. Hart ${ }^{1}$ \\ ${ }^{1}$ University of Illinois at Urbana Champaign, USA \\ ${ }^{2}$ Microsoft Research, USA
}

\begin{abstract}
Panoramic images capture cityscapes of dense urban structures by mapping multiple images from different viewpoints into a single composite image. One challenge to their construction is that objects that lie at different depth are often not stitched correctly in the panorama. The problem is especially troublesome for objects occupying large horizontal spans, such as telephone wires, crossing multiple photos in the stitching process. Thin lines, such as power lines, are common in urban scenes but are usually not selected for registration due to their small image footprint. Hence stitched panoramas of urban environments often include "dented" or "broken" wires. This paper presents an automatic scheme for detecting and removing such thin linear structures from panoramic images. Our results show significant visual clutter reduction from municipal imagery while keeping the original structure of the scene and visual perception of the imagery intact.
\end{abstract}

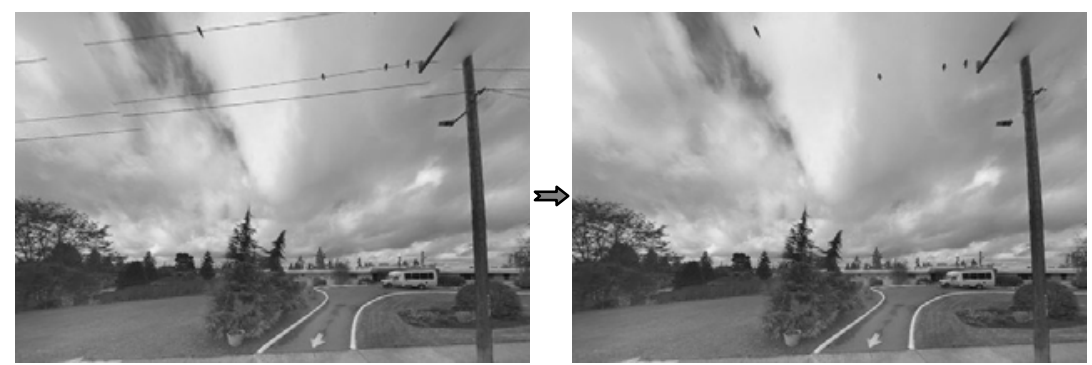

\section{Introduction}

Multi-perspective panoramic imaging produces visual summaries of scenes that are difficult to capture in a camera's limited field of view. As a result, multi-perspective panoramas have seen increasing popularity in navigation and sightseeing consumer applications. For example, Microsoft Street Slide renders multi-perspective panoramas in real time, thus enabling an interactive urban sightseeing experience [12]. We show an example Street Slide urban panorama in Figure 1.

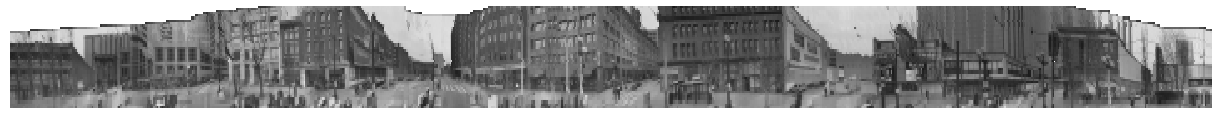

Fig. 1. Panorama of a Long Street [12] 
Until automatic multi-perspective panorama production methods were developed, panorama production typically relied on single-perspective, orthographic projections. In single-perspective panoramas, each point in the world is mapped to the closest point in the panorama's plane [Szeliski 2006]. As a result, single-perspective panoramas suffer from the unnatural effect that far-away objects and close-up objects appear at the same scale [Pulli 2010]. This effect is particular apparent in long panoramas of city streets. Multi-perspective panoramas avoid this unnatural effect by stitching images from disparate viewpoints in a panorama [Rav-Acha]. Each portion of a multi-perspective panorama looks like a natural-perspective view of the scene, though the panorama as a whole does not adhere to a single linear perspective [Agarwala 2006, Vallance 2001].

In the last few years, the computer vision community has made significant strides in automating the production of multi-perspective panoramas. In 2004, Roman et al. developed a system that relied on some human interaction to produce multiperspective panoramas [Roman et al. 2004]. By 2006, Roman and Lensch succeeded in automating this process [Roman and Lensch 2006]. Automatic multi-perspective panorama production involves stitching images together along seams that best merge overlapping features [Szeliski 2006]. Toward this goal, stitching techniques prioritize large objects with low depth variances (such as building facades), isolated objects, and objects with small horizontal spans (such as poles and people). However, smaller objects that lie at a different depth can confound stitching, and appear broken or multiple times in the panorama. In Fig.2 (Top), the panoramic image shows a smooth stitching of the facades, but power lines which are at different depths are distorted. Fig. 2 (Bottom) demonstrates how removing linear clutter such as power lines enhances the quality of panoramas.

We present a novel method for the automatic removal of linear clutter from multiperspective panoramas. Our method focuses on the removal of linear features that are situated in front of high-contrast backgrounds, such as power lines in front of the sky. Our method uses a modified Hough transform to detect problematic thin horizontal features. We remove unwanted horizontal features with a short linear filter. These steps form a method that improves the appearance of automatically constructed panoramas. Our method also reduces the amount of user intervention needed for the construction of high-quality multi-perspective imagery.

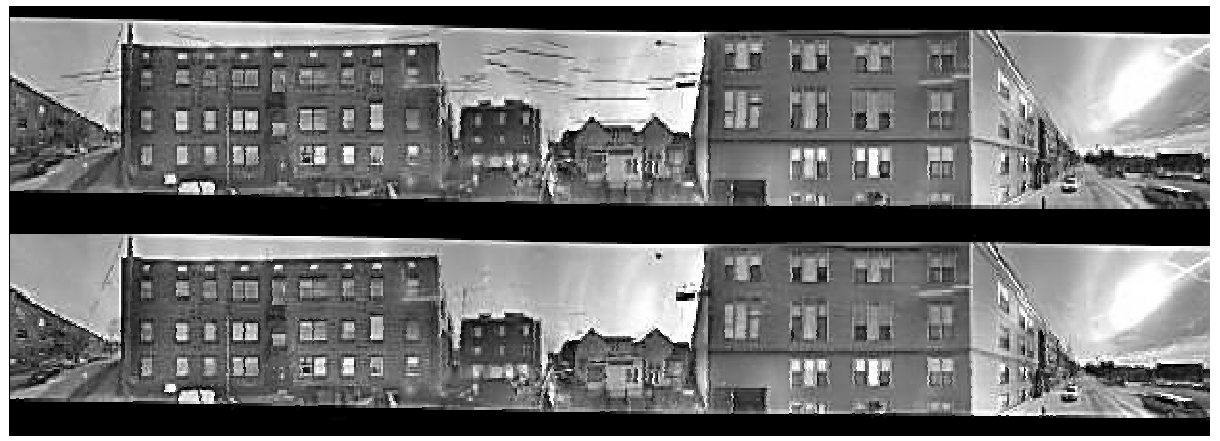

Fig. 2. (Top) Panorama stitched from a group of images taken along a street, including horizontal line multi-perspective stitching artifacts caused by power lines. (Bottom) The same scene where power line artifacts are removed. (Note: We didn't intend to remove close to vertical lines) 


\section{Background}

Methods for automatically detecting and removing wires from images have been developed for outdoor power line inspection and for the cinema special effects industry. In this section, we place our work in the context of past wire detection and removal methods. We also discuss limitations of past work, and we explain how our method overcomes these limitations.

In collaboration with power line maintenance companies, two computer vision studies present methods for detecting power lines in aerial images. These studies enable the use of using small airplanes for inspecting outdoor power lines. Yan et al. apply a Radon transform to extract line segments from power lines in aerial images [24]. Next, Yan et al. use a grouping method to link the line segments and a Kalman filter to connect the detected segments into an entire line. Mu et al. extract power lines from aerial images with a Gabor filter and a Hough transform [13].

The studies by Yan et al. and $\mathrm{Mu}$ et al. make the simplifying assumption that power lines are perfectly straight $[13,24]$.These studies also assume that power lines are made out of a special metal, which has a uniform width and brightness. In contrast, our method breaks image regions into small linear parts that allow power lines to curve, and rely on contrast but not constant color along the line. Therefore, our method succeeds in detecting linear clutter artifacts with varying width and brightness. Also, unlike these power line detection methods, our method both detects and removes the linear clutter from images.

Hirani and Totsuka developed a method for removing linear clutter from video frames $[8,9]$.Their method is especially targeted toward the cinema special effects community. The Hirani-Totsuka method succeeds in applications such as removing wires that actors hang from while doing stunts and removing scratches in old film. Hirani and Totsuka achieve linear clutter removal by applying projection onto convex sets (POTS).The method is effective specially for complex backgrounds, but it is not fully automated in users perspective since it requires the user to manually choose the linear clutter regions. In contrast, our linear clutter removal method is fully automated although it solves this need for user intervention by extracting sky regions.

\section{Linear Clutter Detection}

Existing methods for extracting lines from images, such as the methods discussed in Section 2, rely on either the Hough or Radon transform [3,7]. These line detection techniques alone are insufficient for removing telephone and power wires. First, these wires are usually not straight lines and form catenoid curves. Second, current line detection techniques utilize edge detection output, which for a thin line appears as a pair of edge-detector gradient lines on each side of the wire instead of the wire itself. (We illustrate this in our Experimental Results section and in Fig.7.) We customize these edge detection approaches to handle thin, horizontal features. We also consider that the color of the top and bottom neighboring pixels on a linear wire are similar. This criterion further enhances our line detection by making sure the color of the regions on each sides of the line are the same which is contrary to generic edge detection filters. Moreover, we consider that wires can have different diameters so we have to capture them at any width. 

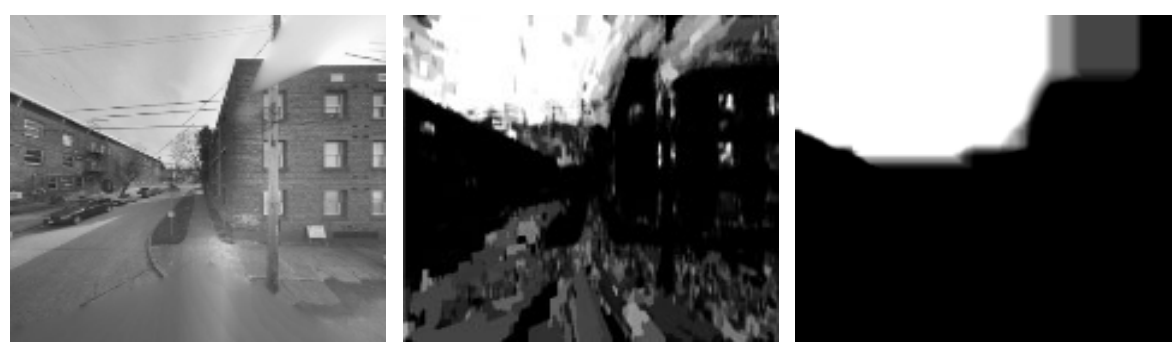

Fig. 3. Finding the sky region of an image. (Left) Original Image. (Center) Sky Confidence Map. (Right) Our Refined Sky Confidence Map.

Due to the visual complexity of building structures, we are less interested in removing lines from front of buildings facades, and focus primarily on thin horizontal occlusions of the sky region. Building façade structures have complex textures that themselves often contain many horizontal lines (like window separators and bricks). We seek to avoid blurring the fine details of these building textures. Therefore, we focus on the more distracting sky related candidate regions for line removal. Our wire removal algorithm first identifies the region of the image corresponding to the sky, and then tracks and removes thin linear features within this region.

We first characterize the sky. Using the input images for the panorama, we find sky related pixels using a depth map if available [2], SkyFinder [20], or scene interpretation [10]. We then create a 2-D (HxS) histogram of the hue and saturation values of the pixels detected as "sky," and selecting the most popular hue/saturation combinations as the sky color. We illustrate an example sky mask in Fig.3.

We then construct a sky mask, where each pixel in the mask is its value from the (normalized) sky histogram for that pixel's hue and saturation. The resulting mask will be noisy and contains many small non-sky regions so we filter it using a Gaussian (or edge-preserving bilateral) low-pass smoothing filter, followed by a morphological "opening" operation consisting of erosions followed by dilations to remove features such as windows reflecting the sky.

For extracting the wire confidence map, we convolve the image with a set of different vertical width filters in order to find the pixels that most likely belong to

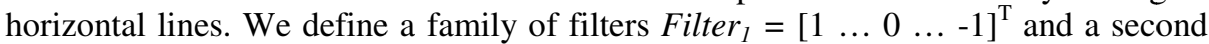
family of filters Filter $_{2}=\left[\begin{array}{lllll}1 & \ldots & -2 & \ldots & 1\end{array}\right]^{\mathrm{T}}$.

Filter $_{1}$ searches for pixels whose top and bottom neighbors are similarly colored. Filter $_{2}$ searches for pixels that are significantly darker than their vertical neighbors. For $512 \times 512$ pixel input images, we observed that the number of pixels in both filters ranges from 3 through 11 (this range is the parameter which users need to provide before running our algorithm).

We compute the quotient Filter $^{l}\left(\mathrm{p}_{i}\right)=\mid$ Filter $_{2}^{l}\left(\mathrm{p}_{i}\right) /$ Filter $_{1}{ }^{l}\left(\mathrm{p}_{i}\right) \mid$ for each filter width $3,5, \ldots, 11$, and for each pixel $\mathrm{p}_{i}$ in the sky region. We show an example application of these filters in Fig.4. For each pixel, we pick the largest absolute value returned from all filter sizes and scale the result by the sky region confidence map, $\operatorname{Filter}\left(p_{i}\right)=$ $\max _{l \in(3,5, \ldots, 11)}\left(\right.$ Filter $\left.^{l}\left(p_{i}\right) \times H S\left(p_{i}\right)\right)$. Two variables called min_line_width and max_line_width (in our example 3 and 11) need to be provided by the user. 

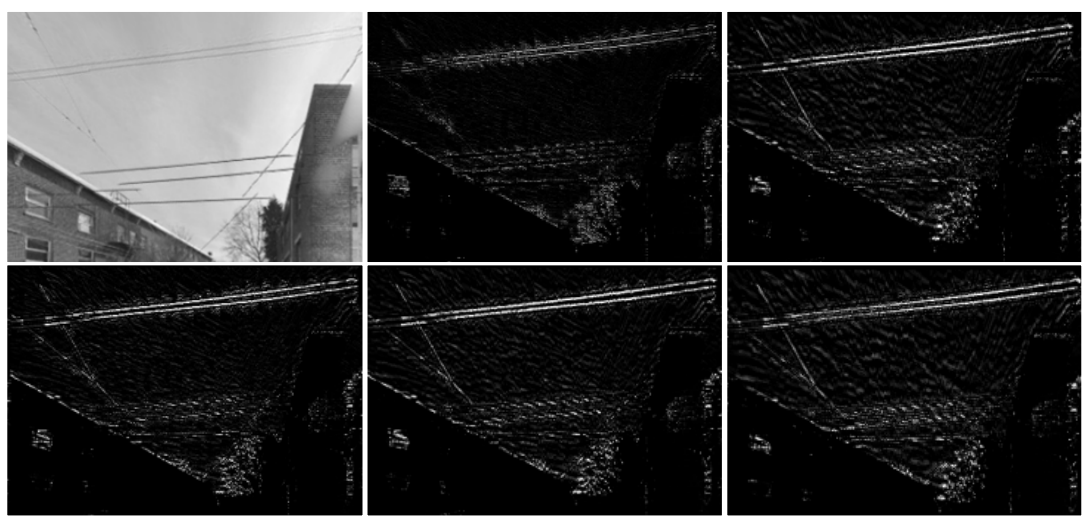

Fig. 4. (Top left) Original image. (Top center, top right, bottom row) Line confidence map for filter widths of $3,5,7,9$ and 11 pixels.

Using a generic Hough transform, some pixels will be detected that don't belong to horizontal lines. We modify the Hough transform to find candidate partial horizontal lines in the image. We remove these pixels by considering the gradient entropy at each pixel. Pixels which belong to a gently curving line should have low gradient direction entropy, so we remove pixels with high gradient direction entropy. This can easily be done by passing a smoothing filter over an image of the gradient tions $\theta=\operatorname{atan}\left(\frac{\text { grad }_{\text {vertical }}}{\text { grad }_{\text {horizontal }}}\right)$ of the input image of potential lines.

We create four bins for angles (0-45), (45-90), (90-135) and (135-180) degrees that are incremented when a pixels gradient falls within that range of directions. If the entropy of a bin is above $80 \%$ of the maximum entropy value for a line (since we have 4 bins the maximum line entropy is about 1.39 [26]) this means this region belongs to a non-consistent gradient (clutter) so we remove it.

Line segments near boundaries of sky regions can be missed by this classifier. Hence, our Hough transform's bins are restricted to horizontal angles from -45 to 45 degrees, and from the peaks of its histogram of line parameters, we find the corresponding pixels in the line image. When these detected lines end near the boundary of the sky region, we extend the line to the boundary. We also break up long line segments into smaller chunks to more accurately represent curved lines.

As illustrated in Fig. 5 right, since we want to eliminate false points on extracted lines, for each pixel in the lines detected by our modified Hough transform, we create a vertical neighborhood (in our case six pixels above and below the line pixel). We then search for the peak contrast pixel in the vertical neighborhood to find the best corresponding point on the line.

For each neighborhood, we find the highest contrast pixel and fit a regression line to its neighboring pixels for each detected line segment. If the variance of the difference between these high-contrast pixels and the regression line exceeds a predefined threshold then we reject the line segment. 


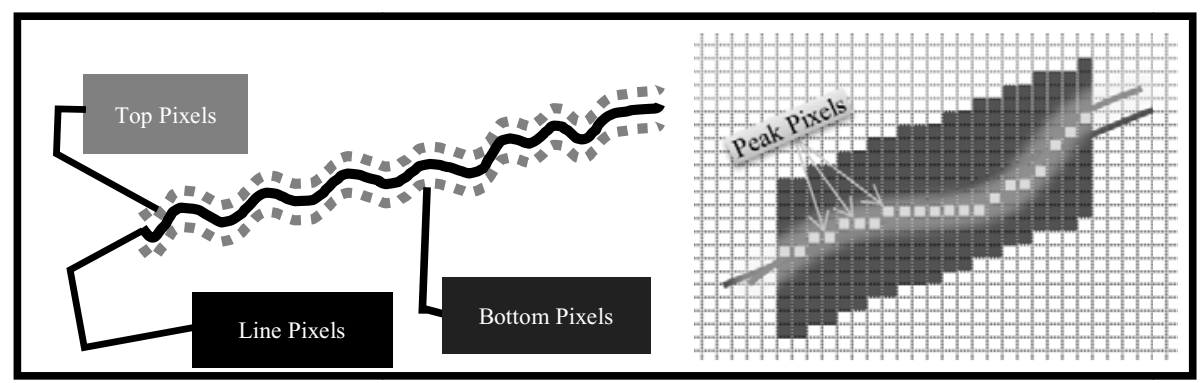

Fig. 5. (Left) Choosing Top and Bottom Pixels of Partial Line. (Right) Finding peak pixels along a line segment.

\section{Linear Clutter Removal}

In this step we pass a bilateral median filter over the image using neighborhood size (max_line_width*3, max_line_width*3), where max_line_width was defined in Section 3. Having found the peak pixels from the previous step, we create a new map consisting of peak pixels and their vertical neighbors within filter_width distance. filter_width refers to the filter size which had the highest return for line detection. We replace each pixel in this new removal map with its median filter image value which was extracted at the beginning of the removal step (Fig. 6).
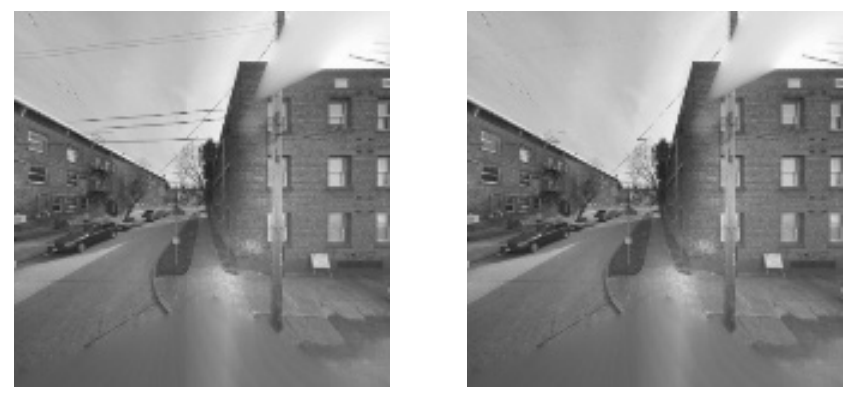

Fig. 6. Blurring (Left) original image. (Right) blurred horizontal wires.

\section{Experimental Results}

We implemented the linear clutter detection and removal algorithm described in Sections 3 and 4 in MATLAB. We tested the performance of each component on a 64 bit, $2.2 \mathrm{GHZ}$ computer. In our tests, we found that calculating the sky mask takes about 0.7 seconds in MATLAB. The subsequent wire detection steps require roughly 12 seconds of runtime per image ( $512 \times 512$ pixels). Blurring the image to remove linear clutter takes less than one tenth of one second. We predict that, if we implement our method in $\mathrm{C}++$ instead of MATLAB, a further performance improvement would 
be easily attainable. As mentioned earlier, the main and most important parameters that are needed for this algorithm are the min and max line width.

One of the most aspects of our method is that is that our unique filter which focuses only on extracting lines which belong to wires on high-contrast backgrounds. Our method avoids extracting edges and linear features on building facades. Fig. 7 demonstrates the advantages of our method over two general edge detection techniques.
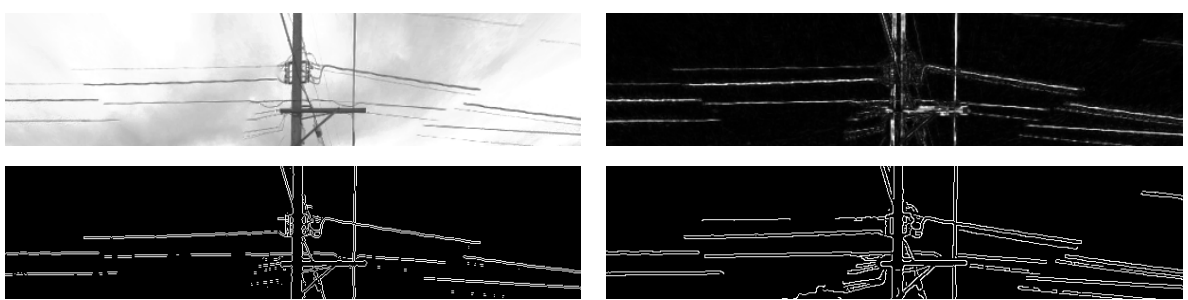

Fig. 7. Doubled lines in edge detection vs. single lines in our method, top-left: original panorama, top-right: our method, bottom-left: sobel, bottom-right: canny

A challenge to our algorithm was that the facades of the buildings which contained big sky colored regions (such as reflection of the sky on the windows) made the rejection fail on those regions and hence, blurred (Fig. 8).
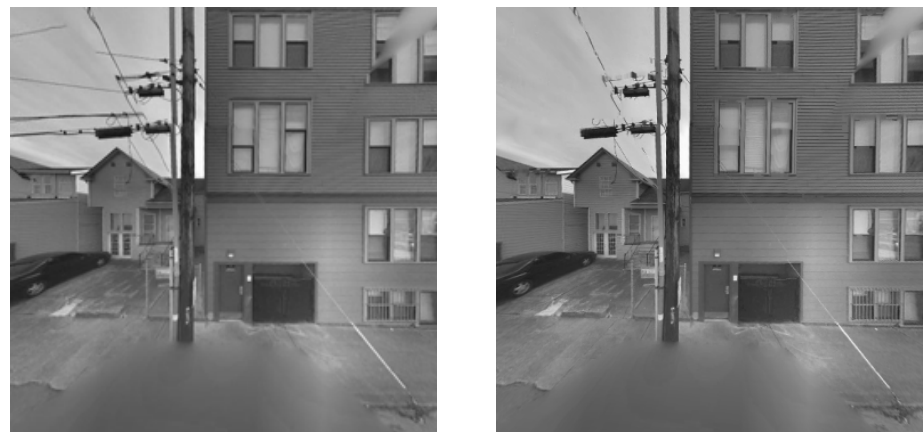

Fig. 8. (Left) Original Image, (Right) linear clutter removal result. Problem is visible on blurred pixels on windows which match sky color and didn't get avoided.

Fig. 9 shows some samples of real urban scene panoramas which their linear clutter has been removed using our technique. As it's visible from the images, the clutter in these panoramas has significantly been reduced.

Another fact to consider on our method is deciding how much blurring trees vs. removing all the visible clutter mattered. This affected how we chose the rejection threshold for gradient entropy. Fig. 10 shows an example of choosing different entropy thresholds. Particularly on the left image, the evergreen tree top is blurred due to the low entropy rejection threshold. 

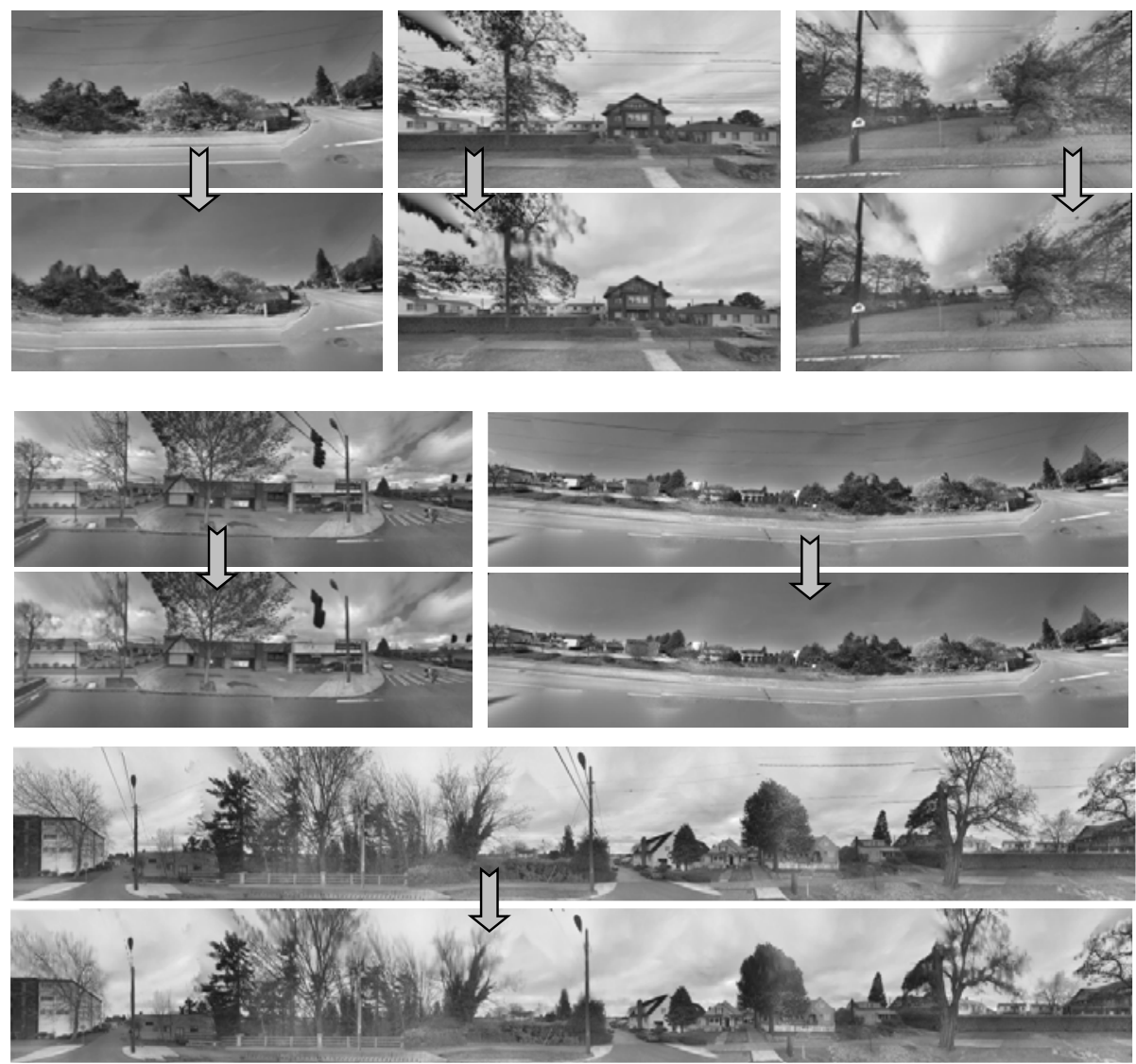

Fig. 9. Experimental Results on Different Urban Panoramas 


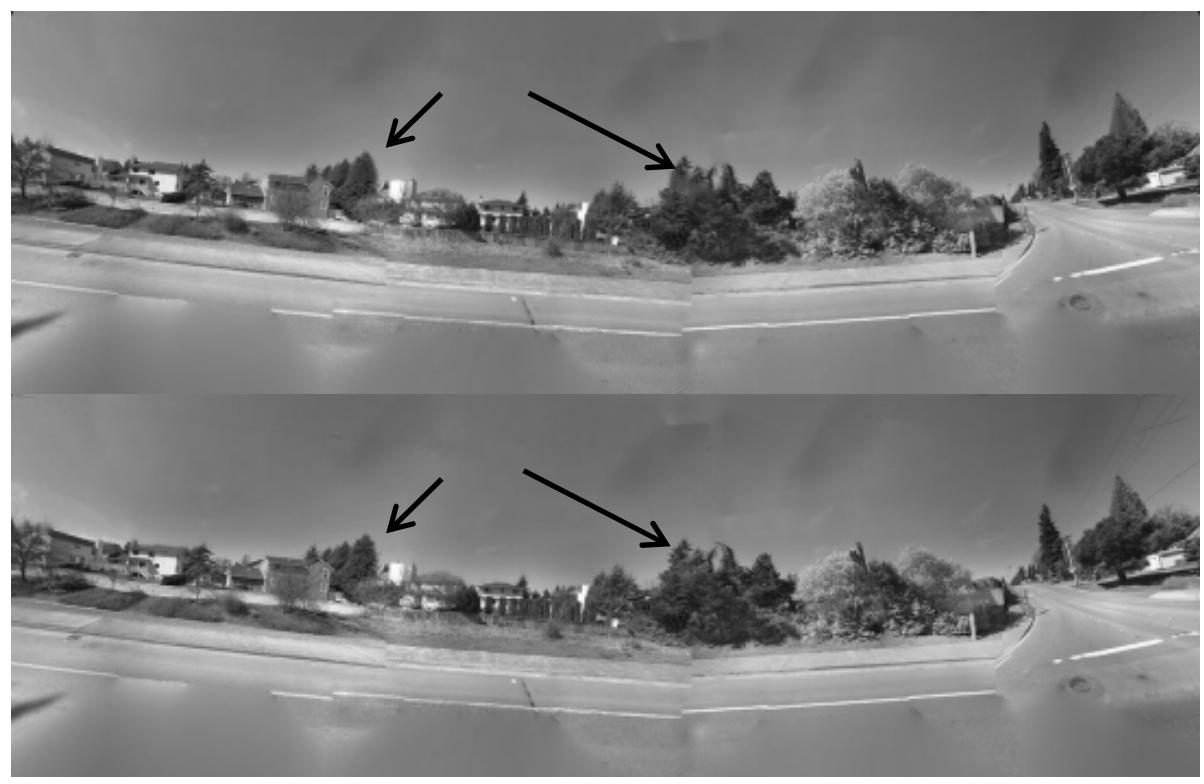

Fig. 10. Effect of different rejection entropy thresholds on blurring the trees. (Left) low threshold (look at the left big evergreen). (Right) high threshold.

\section{Conclusion}

We demonstrated a technique for identifying and removing line clutter from images. This method applies to thin, quasi-horizontal, quasi-linear features that cross the sky. Our technique enhances panoramic scenes that contain power lines or other linear clutter. In future we could take a look at replacing the removed lines with clean Bezier curve replacements and synthetic telephone lines in order to create an exact match to the original scene. Our technique is already being integrated into a well-known urban navigation application.

\section{References}

1. Agarwala, A., Agrawala, M., Cohen, M., Salesin, D., Szeliski, R.: Photographing long scenes with multi-viewpoint panoramas. ACM Trans. Graph 25, 853-861 (2006)

2. Battiato, S., et al.: 3D stereoscopic image pairs by depth-map generation. In: Symposium on 3D Data Processing, Visualization, and Transmission (2004)

3. Beylkin, G.: Discrete radon transform. IEEE Trans. Acoustics, Speech, and Signal Processing 35, 162-172 (1987)

4. Blazquez, C.H.: Detection of problems in high power voltage transmission and distribution lines with an infrared scanner/video system. In: SPIE, pp. 27-32 (1994)

5. ColorPilot. Retouch Unwanted Objects on Your Photos (2011), http: / /www.colorpilot.com/wire.html

6. Fu, S.Y., et al.: Image-based visual servoing for power transmission line inspection robot. International J. of Modelling, Identification and Control 6, 239-254 (2009) 
7. Ginkel, M.V., Hendriks, C.L., Vliet, L.J.: A short introduction to the Radon and Hough transforms and how they relate to each other. Delft University of Technology Technical Report (2004)

8. Hirani, A., Totsuka, T.: Projection Based Method for Scratch and Wire Removal from Digital Images. United States Patent US 5974194 (1996)

9. Hirani, A.N., Totsuka, T.: Combining frequency and spatial domain information for fast interactive image noise removal. In: SIGGRAPH, pp. 269-276 (1996)

10. Hoiem, D., Efros, A., Herbert, M.: Closing the loop in scene interpretation. In: IEEE Conf. on Computer Vision and Pattern Recognition (CVPR), pp. 1-8 (2008)

11. Kent, B.: Automatic Identification and Removal of Objects in Image Such as Wires in a Frame of Video. United States Patent Application US 208, 053 (2008)

12. Kopf, J., Chen, B., Szeliski, R., Cohen, M.: Street slide: browsing street level imagery. ACM Trans. Graph 29 (2010)

13. $\mathrm{Mu}, \mathrm{C}$. , Yu, J., Feng, Y., Cai, J.: Power lines extraction from aerial images based on Gabor filter. In: SPIE (2009)

14. Pulli, K., Tico, M., Xiong, Y.: Mobile panoramic imaging system. In: CVPRW, pp. 108115 (2010)

15. Rav-Acha, A., Engel, G., Peleg, S.: Minimal Aspect Distortion (MAD) Mosaicing of Long Scenes. International J. of Computer Vision 78, 187-206 (2007)

16. Roman, A., Garg, G., Levoy, M.: Interactive design of multi-perspective images for visualizing urban landscapes. IEEE Visualization, 537-544 (2004)

17. Roman, A., Lensch, H.P.: Automatic Multiperspective Images. In: Eurographics Symposium on Rendering Techniques, pp. 83-92 (2006)

18. Seymour, M.: The Art of Wire Removal (2007), http: / /www. fxguide.com/article453.html

19. Szeliski, R.: Image Alignment and Stitching: A Tutorial. Foundations and Trends in Computer Graphics and Vision 2, 1-104 (2006)

20. Tao, L., Yuan, L., Sun, J.: SkyFinder: Attribute-based Sky Image Search. ACM Trans. Graph. 28 (2009)

21. Tomasi, C., Manduchi, R.: Bilateral filtering for gray and color images. In: IEEE International Conf. on Computer Vision, ICCV (1998)

22. Vallance, S.: Multi-perspective images for visualisation. In: Pan-Sydney Area Symposium on Visual Information Processing, VIP (2001)

23. Xiao, Z.: Study on methods to extract transmission line information from high-resolution imagery. In: SPIE (2009)

24. Yan, G., et al.: Automatic Extraction of power lines from aerial images. IEEE Geoscience and Remote Sensing Letters 4, 387-391 (2007)

25. Zuta, M.: Wire Detection System and Method. United States Patent US 6278409 (2001)

26. Rheingold, H.: Tools for Thought: The History and Future of Mind-Expanding Technology, ch.6. The MIT Press, Redmond (2000) 